\title{
EDITORIAL
}

\section{DUAZARY: SUS PROCESOS Y AVANCES EDITORIALES}

\author{
Guillermo Augusto Ceballos-Ospino ${ }^{1}$, Oskarly Pérez-Anaya ${ }^{2}$ \\ 1. Editor - Universidad del Magdalena, Colombia. Correo: guillermoceballos@gmail.com - http://orcid.org/0000-0002-1568-7058 \\ 2. Asistente editorial- Universidad del Magdalena, Colombia. Correo: oskperez123@gmail.com - http://orcid.org/0000- \\ 0002-0701-7847
}

La revista Duazary surgió en el 2004 y en ella se publica información científica sobre temas de salud, desde perspectivas amplias y generales de la salud pública, hasta temas especializados y concretos de áreas clínicas. Ha sido clasificada en categoría C por Publindex-Colciencias ininterrumpidamente a partir del año 2007 hasta la actualidad; así mismo, se encuentra indexada en más de treinta bases, directorios, repositorios e índices entre los que se destacan $C A B$ Abstracts, Emerging Sources Citation Index e Index Copernicus. De esta última base vale la pena destacar que entre más de 25 revistas colombianas que se postularon el año pasado, Duazary fue una de las seis aceptadas. De la misma forma, en el reciente Ranking publicado por Sapiens Research, esta subió dos categorías y se ubicó en la D6. Adicionalmente, la revista llega a una gran parte de nuestro país, a varios países de Latinoamérica y a España, debido a que muchos de los autores de los artículos publicados son oriundos bien de este país, bien de uno perteneciente a la región latinoamericana.

Por lo anterior, y como parte del mejoramiento constante que se viene presentando en la revista, se le ha acuñado un nuevo subtítulo que se visualiza desde la edición 2017-1; este es "Duazary: Revista Internacional de Ciencias de la Salud".

Es un orgullo para la Facultad de Ciencias de la Salud, a la cual pertenece la revista, ver que en la Web de Google

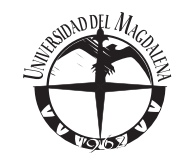

COLCIENCIAS, a pesar de que el panorama que muestra esta entidad para las revistas colombianas es caótico².

Otro punto importante que destacar son las investigaciones publicadas en el último quinquenio, las cuales han recibido un número considerable de citas que han contribuido al posicionamiento de la revista. Entre estos estudios se pueden resaltar: el del psicólogo educativo ${ }^{3}$, el de estilos de vida, el de ideación suicida, el de actividad sexual y calidad del dormir en estudiantes adolescentes y adultos ${ }^{4-9}$, el de bullying: un fenómeno en trasformación ${ }^{10}$, el de la discapacidad en la legislación colombiana ${ }^{11}$, el del suicidio de féminas en el departamento del Magdalena ${ }^{12}$, el del conocimiento de las madres en enfermedades virales ${ }^{13}$ y el de la depresión en pacientes pediátricos con cáncer ${ }^{14}$.

Por último, es un deber de la revista agradecer el apoyo que la Universidad del Magdalena le ha brindado a la misma durante sus años de existencia y los nuevos planes que tiene para fortalecerla.

\section{REFERENCIAS BIBLIOGRÁFICAS}

1. Google scholar. [Consultado el 17 de enero de 2017]. Disponible en: https://scholar.google.es/ scholar?hl $=$ es\&as_sdt $=0,5 \& q=$ revista + duazary

2. PUBLINDEX - COLCIENCIAS. Política nacional para mejorar el impacto de las publicaciones científicas nacionales, Documento $\mathrm{N}^{\circ}$ 1601. Dirección de Fomento a la Investigación. 2016 Aug.

3. Arvilla-Ropain AR, Palacio-Orozco LP, ArangoGonzáles CP. El psicólogo educativo y su quehacer en la institución educativa. Duazary. 2011; 8(2):258-61. 
4. Ferrel F, Ortiz A, Forero L, Herrera M, Peña Y. Estilos de vida y factores sociodemográficos asociados en adolescentes escolarizados del distrito de Santa Marta, Colombia. Duazary. 2014 Dic; 11(2):105-14.

5. Monterrosa A, Ulloque L, Carriazo S. Calidad del dormir, insomnio y rendimiento académico en estudiantes de medicina. Duazary. 2014 Dic; 11(2):85-97.

6. Ceballos-Ospino G, Suarez-Colorado Y, SuescúnArregocés J, Gamarra-Vega L, Gonzalez K, SoteloManjarres A. Ideación suicida, depresión y autoestima en escolares de Santa Marta. Duazary. 2015 jun; 12(1):15-22.

7. Díaz CE, Rodríguez W, Lara L, Matute R, Ariza H. Factores asociados al ejercicio de la sexualidad de las y los adolescentes escolarizados de Cartagena-Colombia 2008. Duazary. 2014 Dic; 10(2):88-97.

8. Martínez J, Maya N, Parada L, Duarte K. Diferencias de las prácticas sexuales entre hombres y mujeres, en estudiantes universitarios durante el primer semestre de 2013. Duazary Dic; 10(2):112-8.

9. Suárez-Colorado Y, Guzmán-García K, Medina-Alfonso L, Ceballos-Ospino G. Características de inteligencia emocional y género en estudiantes de psicología y administración de empresas de una universidad pública de Santa Marta, Colombia: un estudio piloto. Duazary. 2012; 9(2):132-9.

10. Rodríguez-Díaz A, Mejía-Moreu Y. Bullying: un fenómeno por transformar. Duazary. 2012; 9(1): 98-104.

11. Martínez-Rozo A, Uribe-Rodríguez A, VelázquezGonzález H. La discapacidad y su estado actual en la legislación colombiana. Duazary. 2015 jun; 12(1):49-58.

12. Pérez-Manjarrez D, Calderón-Araujo A. Prevalencia del suicidio femenino en el departamento del Magdalena (Colombia): 2004-2010. Duazary. 2012; 9(1):87-93.

13. Lapeira P, Salazar D. Conocimientos y prácticas sobre enfermedades diarreicas y respiratorias entre madres de una institución de salud. Duazary. 2014 Jun;11(1):36-40.

14. Velásquez-Silva S, Zuluaga-Sarmiento L. Factores relacionados con la depresión en pacientes pediátricos con cáncer y participación de la enfermera en su detección. Duazary. 2015 dic; 12(2):164-73. 